1 Carbohydrate gel ingestion significantly improves the intermittent endurance capacity, but not sprint performance, of adolescent team games players during a simulated team games protocol.

4

\title{
5 Authors:
}

6 Shaun M. Phillips, Anthony P. Turner, Mark F. Sanderson \& John Sproule.

8 Affiliation:

9 Institute of Sport, Physical Education and Health Sciences, University of Edinburgh

\section{Correspondence:}

12 Mr Shaun Phillips

13 Institute of Sport, Physical Education and Health Sciences

14 University of Edinburgh

15 St Leonards Land

16 Holyrood Road

17 Edinburgh

18 EH8 8AQ

19 UK

20 Tel: $+44(0) 1316509788$

21 Fax: $+44(0) 1316516521$

$22 \quad$ S.M.Phillips@sms.ed.ac.uk

25 Running Title: Carbohydrate gel and team games performance. 


\section{Abstract}

3 The aim of this study was to investigate the influence of ingesting a carbohydrate (CHO) gel

4 on the intermittent endurance capacity and sprint performance of adolescent team games

5 players. Eleven participants (mean age $13.5 \pm 0.7$ years, height $1.72 \pm 0.08 \mathrm{~m}$, body mass

6 (BM) $62.1 \pm 9.4 \mathrm{~kg}$ ) performed two trials separated by 3-7 days. In each trial, they

7 completed four 15 min periods of part A of the Loughborough Intermittent Shuttle Test,

8 followed by an intermittent run to exhaustion (part B). In the 5 min pre-exercise, participants

9 consumed $0.818 \mathrm{ml} \cdot \mathrm{kg}^{-1} \mathrm{BM}$ of a $\mathrm{CHO}$ or a non-CHO placebo gel, and a further $0.327 \mathrm{ml}^{\mathrm{kg}} \mathrm{kg}^{-}$

$10{ }^{1} \mathrm{BM}$ every $15 \mathrm{~min}$ during part A of the LIST $\left(38.0 \pm 5.5 \mathrm{~g} \mathrm{CHO} \cdot \mathrm{h}^{-1}\right.$ in the CHO trial).

11 Intermittent endurance capacity was increased by $21.1 \%$ during part B when the $\mathrm{CHO}$ gel

12 was ingested $(4.6 \pm 2.0$ vs. $3.8 \pm 2.4 \mathrm{~min}, P<0.05, r=0.67)$, with distance covered in part B

13 significantly greater in the CHO trial ( $787 \pm 319$ vs. $669 \pm 424 \mathrm{~m}, P<0.05, r=0.57)$. Gel

14 ingestion did not significantly influence mean $15 \mathrm{~m}$ sprint time $(P=0.34)$, peak sprint time

$15(P=0.81)$, or heart rate $(P=0.66)$. Ingestion of a $\mathrm{CHO}$ gel significantly increases the

16 intermittent endurance capacity of adolescent team games players during a simulated team

17 games protocol.

18

19

20

21

22

23 Key Words: performance; nutrition; supplement; young people; LIST 
3 We have previously demonstrated a significant enhancement in the intermittent endurance

4 running capacity (hereafter referred to as intermittent endurance capacity) of 12-14 year old

5 team games athletes with ingestion of a 6\% carbohydrate-electrolyte (CHO-E) solution

6 during a modified Loughborough Intermittent Shuttle Test (LIST, Phillips et al 2010). This

7 was achieved with a mean carbohydrate (CHO) intake of $\sim 35 \mathrm{~g} \cdot \mathrm{h}^{-1}$, or $0.78 \mathrm{~g} \cdot \mathrm{kg}^{-1}$ body mass

8 (BM), which is notably lower than the CHO intake in related adult work ( 60-92 g.h ${ }^{-1}$,

9 Foskett et al 2008; Nicholas et al 1995; Welsh et al 2002). Young people display a different

10 metabolic response to exercise than adults, characterised by an enhanced rate of fat

11 metabolism and attenuated rate of endogenous $\mathrm{CHO}\left(\mathrm{CHO}_{\text {endo }}\right)$ use (Riddell 2008; Timmons

12 et al 2007), perhaps to preserve endogenous glycogen stores, which may be lower than adults

13 (Aucouturier et al 2008). Despite this, young people appear able to oxidise exogenous CHO

$14\left(\mathrm{CHO}_{\text {exo }}\right)$ at $\mathrm{BM}-$-relative rates equal to, or greater than, adults for the same relative $\mathrm{CHO}$

15 ingestion rate (Timmons et al 2003). Therefore, $\mathrm{CHO}_{\text {exo }}$ requirements of young people may

16 be different to those of adults, perhaps with a lower rate of $\mathrm{CHO}$ ingestion facilitating

17 exercise enhancement (Phillips et al unpublished data). Coupled with the significant

18 enhancement in intermittent endurance capacity reported in our previous study (Phillips et al

19 2010), this knowledge emphasises that findings from studies supplementing $\mathrm{CHO}$ during

20 simulated team games in adults cannot be confidently applied to young people, and a greater

21 research output into $\mathrm{CHO}$ supplementation during simulated team games in adolescents is

22 therefore warranted.

24 In recent years, ingestion of $\mathrm{CHO}$ in the form of a gel has become more prevalent (Havemann

25 et al 2008), due in part to the ability to manipulate $\mathrm{CHO}$ and fluid intake independently, and 
1 to consume greater amounts of $\mathrm{CHO}$, in gel compared with solution form (Pfeiffer et al

2 2010). Carbohydrate gel ingestion has been shown to improve prolonged steady-state cycling

3 performance (Campbell et al 2008; Earnest et al 2004), with a negligible effect on half-

4 marathon running performance (Burke et al 2005). However, the $\sim 2.4 \% \mathrm{BM}$ loss during the

5 run, along with the presence of an order effect for performance time, may have negated the

6 effect of the gel. Specific to team games exercise, Patterson and Gray (2007) showed a 45\%

7 improvement in intermittent endurance capacity when adult male soccer players ingested a

8 CHO gel before and during the LIST. This is comparable to the improvement seen when

9 consuming CHO-E solutions during the same or similar protocol ( $33-52 \%$, Foskett et al

10 2008; Nicholas et al 1995; Welsh et al 2002). To date, this remains the only study to

11 investigate $\mathrm{CHO}$ gel supplementation during team games exercise.

13 The combination of our previous findings regarding the efficacy of a CHO solution during

14 simulated team games in adolescents and the significant improvement in intermittent

15 endurance capacity with $\mathrm{CHO}$ gel ingestion shown by Patterson \& Gray (2007) suggests that

16 ingestion of $\mathrm{CHO}$ gels during simulated team games may benefit adolescent team games

17 players. Therefore, the aim of this study was to determine the influence of ingesting a CHO

18 gel immediately before, and during, a simulated team games protocol on the intermittent

19 endurance capacity and sprint performance of adolescent team games players. It was

20 hypothesised that ingestion of the $\mathrm{CHO}$ gel would significantly improve intermittent

21 endurance capacity, with no significant influence on sprint performance. 


\section{Methods}

3

4

5 Eleven team games players (10 males and 1 female; mean age $13.5 \pm 0.7$ years, height $1.72 \pm$

$6 \quad 0.08 \mathrm{~m}, \mathrm{BM} 62.1 \pm 9.4 \mathrm{~kg}$ ) participated in the study. Participants were recruited from local

7 schools and sports clubs. Inclusion criteria was to be between the ages of 12-14 years,

8 regularly participating in competitive soccer, rugby or field hockey to at least club level, free

9 from any muscle or joint injury, and not taking medication that influences the ability to

10 exercise. All participants were in good health at the time of the study, as determined by

11 completion of a pre-study medical questionnaire. Participants' were either frequent or

12 occasional users of $\mathrm{CHO}$ containing sports drinks.

13

14 Prior to inclusion, comprehensive written and verbal explanation of the study was provided to

15 participants and parents, and written parental informed consent was received. Subsequently,

16 written child assent was gained. The study received ethical approval from the University of

17 Edinburgh Ethics Committee.

18

19 Biological maturity status

20

21 Due to ethical and consensual restrictions regarding direct observational assessment of

22 Tanner stages, biological maturity offset was assessed using the established, non-invasive

23 equations of Mirwald et al (2002), as previously described (Phillips et al 2010). For the

24 participants in this study, mean biological maturity offset was +0.94 years (range -1.77 -

$25+2.68$ years). Mean predicted age at peak height velocity for the female participant was 11.3 
1 years and for the male participants was 12.6 years (range $11.8-13.8$ years). This classifies

2 the female participant as an average maturer, and the male participants as early maturers

3 (Baxter-Jones et al 2005).

$5 \quad$ Preliminary Tests

$7 \quad$ Peak Running Velocity

9 All exercise intensities used in the main experimental protocol were based on percentages of 10 peak running velocity $\left(\mathrm{V}_{\text {peak }}\right)$ as determined from a treadmill $\mathrm{V}_{\text {peak }}$ test. This is opposed to the 11 more common calculation of speed based on percentage of $\dot{\mathrm{VO}}_{2 \max }$, and is believed to more 12 accurately reflect physiological demand during team games (Bangsbo 1994). The

13 physiological responses to maximal treadmill and free range running have been reported to be 14 similar (Crouter et al., 2001). Prior to undertaking the $\mathrm{V}_{\text {peak }}$ test, all participants walked at a 15 self-selected speed on the treadmill (Ergo 55, Woodway, Germany) for 2 min, then

16 completed the first four levels of the $\mathrm{V}_{\text {peak }}$ test as described below to familiarise themselves 17 with the treadmill (Lavcanska et al 2005). Following this, participants sat quietly for $10 \mathrm{~min}$ 18 to recover and allow any residual anxiety to dissipate before starting the test.

20 The $\mathrm{V}_{\text {peak }}$ test, adapted from Marino et al (2004), began at $8 \mathrm{~km} \cdot \mathrm{h}^{-1}$ at a gradient of $1 \%$ for 21 one minute, after which the speed was increased by $0.5 \mathrm{~km} \cdot \mathrm{h}^{-1}$ in one-minute increments until 22 the participant indicated that they could not continue despite strong verbal encouragement. A 23 maximal effort was confirmed by observation of subjective symptoms of fatigue (facial

24 flushing, unsteady gait, heavy sweating, hyperpnoea) and attainment of a heart rate (HR) $\geq$ 25195 beats per min (Armstrong 2007). Peak running velocity and maximum $H R\left(H_{\max }\right)$ were 
1 calculated as the highest treadmill velocity maintained for $30 \mathrm{~s}$ and the highest $5 \mathrm{~s}$ average,

2 respectively. After a 15 min seated recovery participants performed $15 \mathrm{~min}$ of the LIST, as

3 described below, to familiarise themselves with the running speeds required and the data

4 collection procedures.

5

6 Experimental Design

8 All participants completed two trials separated by a minimum of three, and maximum of

9 seven, days. During each trial participants consumed either a 100\% maltodextrin CHO gel

10 (CHO trial) or a non-CHO artificially sweetened gel (placebo (PLA) trial), matched for taste,

11 texture, and mouth-feel (High5 Ltd, Bardon, Leicestershire). All trials were randomised,

12 counterbalanced, and double-blinded to control for order effects and experimenter bias. Each

13 participant completed both trials at the same time of day, or as close to this as possible. The

14 gel was consumed in amounts that enabled a standardised CHO intake of $0.78 \mathrm{~g} . \mathrm{kg}^{-1} \mathrm{BM}$ for

15 each participant, to enable a direct comparison with previous work from this laboratory

16 (Phillips et al 2010). As the two gels were of slightly different colour they were prepared in

17 non-transparent bottles by the individual in control of trial blinding, so that neither the

18 investigator nor the participants could see the gel at any time. Participants were requested to

19 refrain from heavy physical activity for $48 \mathrm{~h}$ before each trial. Additionally, they were asked

20 to record their food and fluid intake, including the portion size of all food consumed and the

21 volume of all fluid ingested, for $24 \mathrm{~h}$ before the first trial. This diet was replicated prior to

22 trial two to standardise muscle and hepatic glycogen concentrations and hydration status.

23 Participants were not requested to record food and fluid intake in the depth of detail that

24 would have enabled a subsequent dietary composition analysis. Requesting this would have

25 placed greater stress on extremely time-pressured participants and their parents, and may 
1 have negatively affected adherence to the dietary record, and/or retention of participants

2 through the full study.

4 Experimental Protocol

6 Standing height was measured using a free-standing adjustable stadiometer (Seca, model no.

7 2251821009, Germany). After voiding and urinating, if necessary, dry nude BM was

8 recorded (Seca Digital, model no. 7052321009, Germany). After attaching the HR monitor

9 chest strap and watch (Polar RS400, Polar Electro Oy, Finland) participants sat quietly for 5

10 min, then completed a standardised warm-up consisting of jogging, striding and dynamic

11 stretching for $10 \mathrm{~min}$. Immediately following the warm-up, participants sat and were

12 instructed to consume the prescribed gel $\left(0.818 \mathrm{ml} . \mathrm{kg}^{-1} \mathrm{BM}\right)$ followed by $5 \mathrm{ml} . \mathrm{kg}^{-1} \mathrm{BM}$ of

13 water during the 5 min before commencing exercise. Water was ingested to offset the

14 potential influence of dehydration (Patterson \& Gray 2007), and was consumed in volumes

15 that have previously been administered in this population during the same exercise protocol

16 (Phillips et al 2010). Once the initial gel and water bolus had been consumed, participants

17 were asked to state which gel they believed was being prescribed.

19 The LIST was conducted indoors, on a level rubber floor, as described elsewhere (Phillips et

20 al 2010). Briefly, participants completed four blocks of part A of the LIST separated by 3

21 min seated recovery, followed by an intermittent run to exhaustion (part B). Participants consumed the solution $\left(0.327 \mathrm{ml} . \mathrm{kg}^{-1} \mathrm{BM}\right)$ followed by water $\left(2 \mathrm{ml} . \mathrm{kg}^{-1} \mathrm{BM}\right)$ in the recovery

23 period between each 15 min block and in the recovery period before commencing part B.

24 After the measurement of post-exercise BM, participants were asked again to state which gel 25 they believed they had received during the protocol, to observe whether exercise exerted any 
1 influence on their gel choice. Participants were clearly informed that they were free to

2 change their mind from their pre-exercise gel choice, or to keep their selection the same.

\section{$4 \quad$ Measurements}

6 Heart rate was recorded at $5 \mathrm{~s}$ intervals throughout the $\mathrm{V}_{\text {peak }}$ test and the experimental

7 protocol using short-range telemetry. Data was retrieved and downloaded onto a computer

8 software program (Polar ProTrainer 5, Polar Electro Oy, Finland) for subsequent analysis.

9 Ambient temperature and humidity were recorded immediately before the start of the

10 protocol and at the end of each 15 min block in part A using a digital hygro-thermometer

11 (Tako Astatic Technology, Malaysia). Ratings of perceived exertion (RPE) were measured

12 during the first shuttle of the final walking phase of each 15 min block in part $\mathrm{A}$ and at

13 exhaustion in part B using the Children's Omnibus Scale of Perceived Exertion (0-10 scale),

14 which has been validated for use with participants of the age range in this study (Roemmich

15 et al 2006). Gut fullness (GF) and gastric discomfort (GD) were assessed immediately on

16 completion of each 15 min block in part A and at exhaustion in part B using anchored 10

17 point scales $(1=$ not at all, $10=$ extremely $)$. Sprint times were measured in one direction by

18 two wireless infrared single-beam photoelectric cells (Speed Trap 2, Gill Athletics, Illinois)

19 placed $15 \mathrm{~m}$ apart. If participants needed to urinate at any time from the onset of the protocol

20 until completion of the measurement of post-exercise BM they did so into a measuring jug,

21 with this volume incorporated into the BM loss calculation. Body mass loss was calculated

22 from the difference between pre- and post-exercise nude BM, corrected for fluid intake and

23 urine output. Sweat rate $\left(\mathrm{SR} ;{\left.\mathrm{L} . \mathrm{h}^{-1}\right)}\right.$ was calculated using the equation: (Pre-exercise BM (kg)

24 + fluid ingested (L) - urine output $(\mathrm{L})$ - post-exercise BM $(\mathrm{kg})$ ) / protocol duration (min) $\mathrm{x}$

2560 (Edwards et al 2007). This calculation does not account for BM loss due to fuel oxidation 
1 and respiratory fluid loss, but it is unlikely these would differ between trials (Edwards et al

2 2007).

$4 \quad$ Statistical Analysis

6 The Shapiro-Wilk test for normality was employed on all data sets. Paired $t$-tests compared

7 between-trials differences in fluid, gel and CHO intake, pre-exercise BM, BM loss and SR,

8 HR during part B, and HR, GF and GD at exhaustion. Distance covered in part A and B,

9 time to exhaustion and RPE at exhaustion were analysed using the Wilcoxon matched-pairs

10 test. Mean ambient temperature and relative humidity, sprint times and peak sprint times,

11 and HR, GF and GD during part A were analysed with a 2 way (gel x time) ANOVA.

12 Bonferroni pairwise comparisons were used to explore significant main effects with the

13 exception of GF, where Wilcoxon matched-pairs tests with Bonferroni correction were used

14 due to the grouped data displaying non-normal distribution. Friedman tests were used to

15 analyse the main effect of time during part A within each trial for RPE. Wilcoxon matched-

16 pairs tests, with Bonferroni correction, explored significant within-trials main effects. Chi-

17 square analysis assessed the frequency distribution of gel choice responses. Effect sizes (ES)

18 from ANOVA were calculated using partial eta squared $\left(\eta^{2}\right)$ values, which were square

19 rooted to give correlation coefficients (Field 2005). Effect sizes generated from $t$ scores were

20 calculated using the equation of Rosnow and Rosenthal (2005), and from $z$ scores using the

21 equation of Rosenthal (1991), to give correlation coefficients (Field 2005). Effect sizes were

22 defined as small $(r=0.1-0.3)$, moderate $(r=0.3-0.5)$, large $(r=0.5-0.7)$, very large $(r=0.7-$

$230.9)$, and nearly perfect $(r=0.9-1.0)$, based on the classifications of Hopkins (2006). Data

24 are mean $\pm \mathrm{SD}$, and with the exception of analyses using the Bonferroni correction,

25 significance was set at $P<0.05$. 
5 Mean $V_{\text {peak }}$ attained in the incremental treadmill run to exhaustion was $14.6 \pm 0.9 \mathrm{~km} \cdot \mathrm{h}^{-1}$.

6 Mean $\mathrm{HR}_{\max }$ and RPE at exhaustion were $197 \pm 6$ beats per min and $9.2 \pm 0.4$, respectively.

Distance covered and time to exhaustion

10 By design, distance covered during part A was the same in the $\mathrm{CHO}$ and PLA trials $(7.1 \pm 0.2$

$11 \mathrm{~km})$. Time to exhaustion during part B of the LIST for both trials is shown in Figure 1.

12 Participants ran for $21.1 \%$ longer in the CHO compared to the PLA trial $(P<0.05, r=0.67)$,

13 corresponding with a significantly greater distance covered in the $\mathrm{CHO}$ trial $(787 \pm 319$ vs.

$14669 \pm 424 \mathrm{~m}, P<0.05, r=0.57)$.

15

The mean time of all sprints, and the mean of participants' peak sprint time only, in each

21 block of part A of the LIST is shown in Figure 2A and 2B, respectively. Sprint times throughout the LIST were faster in the $\mathrm{CHO}$ trial, but did not reach statistical significance

$23\left(F_{1,10}=1.1, P=0.33, r=0.31\right)$. There was also no interaction effect (gel x time, $F_{3,30}=0.4$,

$24 P=0.75, r=0.20)$. There was a main effect of time on sprint duration $\left(F_{3,30}=25.1, P<\right.$

$250.001, r=0.85)$. Sprint times in block 2 were significantly slower than block $1(P<0.05, r=$ 
$10.77)$ and in block 3 were significantly slower than block $2(P<0.05, r=0.80)$. There was

2 no significant difference in sprint time between blocks 3 and $4(P=0.21, r=0.68)$. Mean

3 peak sprint time was not significantly different between-trials $\left(F_{1,10}=0.06, P=0.81, r=\right.$

$40.08)$ and there was no interaction effect $\left(F_{3,30}=0.4, P=0.72, r=0.21\right)$. There was a main

5 effect of time on peak sprint duration $\left(F_{3,30}=15.1, P<0.001, r=0.78\right)$. Peak sprint times in

6 block 3 were significantly slower than block $2(P<0.05, r=0.82)$. There was no significant

7 difference between blocks 1 and $2(P=0.33, r=0.52)$ or 3 and $4(P=1.0, r=0.41)$.

8

\section{PLEASE PLACE FIGURES 2A and 2B HERE}

Heart rate, ratings of perceived exertion, and gastric measures

Mean HR and RPE during part A of the LIST, and mean peak HR and mean RPE at exhaustion in part B are shown in Table 1 . There was no significant treatment $\left(F_{1,8}=0.21, P\right.$ $=0.66, r=0.16)$ or interaction $\left(F_{3,24}=1.34, P=0.29, r=0.38\right)$ effect on HR during part A of the LIST. There was a main effect of time for HR in part $\mathrm{A}\left(F_{1.32,10.56}=12.18, P<0.005\right.$, $r=0.78)$. Heart rate in block 2 was significantly greater than block $1(P<0.001, r=0.95)$. There was no significant difference between blocks 2 and $3(P=1.0, r=0.14)$ or 3 and $4(P$ $=0.97, r=0.35)$. Mean HR during part $\mathrm{B}$ of the LIST was greater in the $\mathrm{CHO}$ trial, but did not reach statistical significance $(175 \pm 5$ vs. $173 \pm 6$ beats per $\min , P=0.42, r=0.31)$. Peak $\mathrm{HR}$ at exhaustion in part B was also higher in the $\mathrm{CHO}$ trial, but again this was not significant $(P=0.56, r=0.23)$. Ratings of perceived exertion were very similar at all time points between trials, with no significant differences found. A main effect of time was present for the $\mathrm{CHO}\left(\chi^{2}(3)=29.8, P<0.001\right)$ and PLA $\left(\chi^{2}(3)=31.1, P<0.001\right)$ trials. Ratings of perceived exertion increased significantly with each successive exercise block $(P<0.001, r=$ 
$10.89,0.76$ and 0.76 , respectively). There was no between-trials difference in RPE at

2 exhaustion $(P=1.0, r=0)$.

3

6 Mean GF and GD during part A of the LIST, and at exhaustion in part B, are shown in Table

7 2. Mean GF was greater in the CHO trial throughout part A of the LIST, but this was not

8 statistically significant $\left(F_{1,10}=3.50, P=0.09, r=0.51\right)$. There was also no interaction effect

$9 \quad\left(F_{1.74,17.36}=0.65, P=0.52, r=0.25\right)$. There was a significant effect of time on GF $\left(F_{1.47,14.71}\right.$

$10=7.72, P<0.01, r=0.66)$. Gut fullness in block 3 was significantly greater than block $2(P$

$11<0.01, r=0.56)$. There was no significant difference between blocks 1 and $2(P=0.06, r=$ $0.40)$ or 3 and $4(P=1.0, r=0)$. Gut fullness scores during part A of the LIST were modest.

13 Gut fullness at exhaustion was higher in the $\mathrm{CHO}$ trial, but this was not statistically

14 significant $(P=0.24, r=0.37)$. There was no treatment $\left(F_{1,10}=0.14, r=0.11\right)$ or interaction

$15\left(F_{3,30}=0.97, r=0.30\right)$ effect on GD. Gastric discomfort increased significantly with time

$16\left(F_{1.45,14.52}=13.06, P<0.005, r=0.75\right)$, and was significantly greater in block 2 than block 1

$17(P<0.05, r=0.64)$ and block 3 than block $2(P<0.05, r=0.54)$. There was no significant

18 difference between blocks 3 and $4(P=1.0, r=0.06)$. Gastric discomfort scores during part

19 A were also moderate. Gastric discomfort at exhaustion was higher in the CHO trial, but this

20 was not statistically significant $(P=0.59, r=0.17)$.

21

PLEASE PLACE TABLE 2 HERE 
3 Mean pre-exercise dry nude BM was not significantly different between the CHO and PLA

4 trials $(62.4 \pm 9.1$ and $62.9 \pm 9.2 \mathrm{~kg}$, respectively, $P=0.27, r=0.34)$. Mean BM loss in the

$5 \mathrm{CHO}$ and PLA trials was $1.0 \pm 0.4$ and $1.1 \pm 0.3 \mathrm{~kg}$, respectively $(P=0.36, r=0.29)$,

6 equating to a mean loss of $1.59 \pm 0.53$ and $1.67 \pm 0.37 \%$ of pre-exercise $\mathrm{BM}(P=0.50, r=$

7 0.22). Mean SR was $0.77 \pm 0.27$ and $0.85 \pm 0.27$ L.h $\mathrm{h}^{-1}$ in the CHO and PLA trials,

8 respectively $(P=0.30, r=0.33)$, equating to a BM-relative mean sweat loss of $12.42 \pm 4.16$

9 and $13.44 \pm 3.70 \mathrm{ml} \cdot \mathrm{kg}^{-1} \mathrm{BM} \cdot \mathrm{h}^{-1}$, respectively $(P=0.39, r=0.28)$.

\section{$11 \quad$ Blinding}

13 After consuming the initial gel bolus immediately prior to exercise, four participants (36\%)

14 correctly identified both gels and seven (64\%) failed to do so. Chi square analysis of the

15 responses in the $\mathrm{CHO}$ trial found a non-significant deviation from the expected response

16 frequency $\left(\chi^{2}(1)=0.818, P=0.37\right)$. Post-exercise, only two participants correctly guessed

17 both gels. In the PLA trial, five participants (46\%) correctly guessed the PLA gel post-

18 exercise when they had guessed incorrectly prior to exercise. In the $\mathrm{CHO}$ trial, no

19 participants correctly guessed the $\mathrm{CHO}$ gel post-exercise after guessing incorrectly pre-

20 exercise, but five participants (46\%) incorrectly chose the PLA gel post-exercise, having

21 guessed correctly pre-exercise. 
3 Mean fluid intake was $811 \pm 119$ and $811 \pm 120 \mathrm{ml}$ for the CHO and PLA trials, respectively

$4 \quad(P=0.93, r=0.03)$. Mean gel intake in the $\mathrm{CHO}$ trial was $132.6 \pm 19.4 \mathrm{ml}$ and in the PLA

5 trial was $132.7 \pm 19.6 \mathrm{ml}(P=0.92, r=0.03)$. Combined fluid and gel intake was $943.6 \pm$

6138.3 and $943.9 \pm 139.4 \mathrm{ml}(P=0.92, r=0.03)$ in the CHO and PLA trials, respectively. In

7 the $\mathrm{CHO}$ trial, total $\mathrm{CHO}$ intake was $38.0 \pm 5.5 \mathrm{~g} \cdot \mathrm{h}^{-1}$, or $0.78 \mathrm{~g} \cdot \mathrm{kg}^{-1} \mathrm{BM}$.

8

9 Ambient temperature and relative humidity

11 Mean ambient temperature and relative humidity during the LIST are shown in Table 3.

12 Mean ambient temperature was not significantly different between $\left(F_{1,10}=3.59, P=0.09, r=\right.$

$130.51)$ or within $\left(F_{1.06,10.58}=0.32, P=0.60, r=0.18\right)$ trials. However, a significant gel $\mathrm{x}$ time 14 interaction was present $\left(F_{1.67,16.70}=3.87, P<0.05, r=0.53\right)$. Mean relative humidity was

15 not significantly different between $\left(F_{1,10}=3.89, P=0.05, r=0.57\right)$ or within $\left(F_{1.9,18.9}=1.38\right.$, $16 P=0.28, r=0.35)$ trials, and there was no interaction effect $\left(F_{1.7,17.4}=1.08, P=0.35, r=\right.$ $17 \quad 0.31)$

18 
3 This is the first study to demonstrate that ingestion of a $\mathrm{CHO}$ gel immediately before, and

4 during, a simulated team games protocol significantly improves the intermittent endurance

5 capacity of adolescent team games players. Carbohydrate gel ingestion did not significantly

6 influence sprint performance during the protocol.

$8 \quad$ Time to exhaustion

10 The $21.1 \%$ improvement in intermittent endurance capacity in the current study is similar to

11 the $24.4 \%$ improvement we recently demonstrated when adolescent team games players

12 ingested equal BM-relative amounts of $\mathrm{CHO}$ via a $6 \%$ CHO-E solution before and during the

13 same exercise protocol (Phillips et al 2010). Therefore, the current study serves to increase

14 the knowledge base and provide further evidence for an ergogenic effect of $\mathrm{CHO}$

15 supplementation during simulated team games in adolescents. It could be inferred that the

16 influence of $\mathrm{CHO}$ reported here may translate into actual team games performance by

17 enabling participants to continue performing high-intensity exercise for longer, which is a

18 recognised marker of performance and fatigue during team games (Carling et al 2008).

19 Using self-paced protocols, such as that proposed by Ali et al (2009), may enable further

20 quantification of this.

21

22 It appears that $\mathrm{CHO}$ gels and solutions of similar composition, when administered in

23 volumes that deliver an equal amount of BM-relative $\mathrm{CHO}$, have similar efficacies for

24 adolescents during simulated team games. The observation of a similar time-course of $\mathrm{CHO}$

25 oxidation and peak $\mathrm{CHO}$ oxidation rate between $\mathrm{CHO}$ gels and drinks of the same 
1 composition (Pfeiffer et al 2010) may help to explain this, but would need to be confirmed in

2 adolescents.

4 The current findings mimic those of Patterson and Gray (2007), who found a 45\%

5 improvement in time to exhaustion at the end of the LIST in the only study to investigate

6 CHO gel supplementation during simulated team games in adults. Patterson and Gray (2007)

7 concluded that the increased intermittent endurance capacity was due to CHO-mediated

8 sparing of muscle glycogen during exercise. The current study did not collect data that would

9 enable direct quantification of enhancement mechanisms due to ethical and consensual

10 restrictions regarding the employment of blood sampling and muscle biopsies in adolescents.

11 However, as discussed in our previous study, metabolic distinctions between adolescents and

12 adults exist that also support the hypothesis of muscle glycogen sparing with CHO ingestion

13 in adolescents (Phillips et al 2010). It is crucial that future research manages to overcome

14 ethical and consensual issues and provide data on the metabolic responses of adolescents

15 during simulated team games with and without $\mathrm{CHO}$ ingestion.

17 The $\sim 21 \%$ improvement in time to exhaustion in the current study is notably lower than the

$1845 \%$ improvement reported by Patterson and Gray (2007). Patterson and Gray (2007) used a

19 PLA solution, matched for taste, colour, and temperature, as a comparison to the CHO gel.

20 Despite matching for these criteria, it is possible that participants would still have been aware

21 of whether they were consuming a gel or a solution, due to the different consistencies of these

22 products. Reporting of blinding statistics would have been useful to validate the success of

23 the single-blinding procedures used. In addition, depending on the amount of information the

24 participants were provided with as to the aims and/or expectations of the study, the use of a

25 PLA solution could have raised a significant potential for experimenter bias and/or 
1 participant expectancy that may have greatly impacted the results. This may help to explain

2 the difference in intermittent endurance capacity improvement compared with the current

3 study. However, the different participant populations used in the two studies (adolescents

4 versus adults) could also have contributed to this difference (Phillips et al 2010).

5

\section{Sprint Duration}

8 The finding that $\mathrm{CHO}$ gel supplementation did not significantly improve mean sprint or mean

9 peak sprint performance during the LIST is in line with other work from this laboratory

10 (Phillips et al., 2010), as well as previous adult work (Patterson \& Gray 2007). Potential

11 reasons for the lack of influence of CHO have been discussed previously (Phillips et al 2010),

12 and the reader is referred here for further information.

14 The only significant attenuation in peak sprint time in the current study occurred between

15 blocks 3 and 4, which also corresponded with a significant increase in both GF and GD

16 measures. It may be that this increase in GF and GD inhibited sprint performance, which is

17 further supported by the significant attenuation of mean sprint time over the same period.

18 However, in our previous study, peak sprint time was significantly attenuated in block 3 with

19 no significant corresponding increase in measures of GF or GD (Phillips et al 2010).

20 Therefore, the parallel increases in these measures in the current study may be coincidental.

Heart rate, ratings of perceived exertion, and gastric measures

24 There were no significant between-trials differences for HR response, RPE, or gastric

25 measures. This replicates findings from our previous study (Phillips et al 2010), and from 
1 related adult work (Ali et al 2007; Nicholas et al 1995; Welsh et al 2002). In our previous

2 study, we demonstrated a significantly greater peak HR at exhaustion in the CHO trial. This

3 finding was not replicated in the current study, indicating that it may have been an artefact of

4 the participant population employed in our previous work, rather than a mechanism of

5 enhanced intermittent endurance capacity with $\mathrm{CHO}$ supplementation.

7 The lack of influence of $\mathrm{CHO}$ on RPE reinforces the notion that $\mathrm{CHO}$ supplementation

8 during sub-maximal team games exercise has a negligible effect on the effort perception of

9 adolescents. Along with the HR data, it can therefore be intimated that enhancements in

10 intermittent endurance capacity with $\mathrm{CHO}$ ingestion in these participants are from a

11 metabolic source. Potential causes of the progressive increase in RPE with time are

12 discussed elsewhere (Phillips et al 2010).

13

14 It appears that a $\mathrm{CHO}$ gel of the composition and [CHO] used in this study is tolerated as well

15 as an isoenergetic $\mathrm{CHO}-\mathrm{E}$ solution by adolescents during simulated team games exercise

16 (Phillips et al 2010). The greater concentration of the gel means that a lower volume is

17 ingested to achieve a given $\mathrm{CHO}$ intake compared with a solution, which may explain the

18 good tolerance of the gels (Noakes et al 1991). Potential reasons for the influence of time on

19 gastric variables have been discussed previously (Phillips et al 2010).

20

21 Carbohydrate and fluid intake

22

23 As evidenced from the above discussion and the results of our previous study (Phillips et al

24 2010), CHO gels and solutions providing the same amount of CHO appear similarly

25 efficacious for maintaining physiological function and improving intermittent endurance 
1 capacity during team games exercise in adolescents. Clearly, the BM-relative and absolute

$2 \mathrm{CHO}$ intake in our two studies is lower than adult research. It is also lower than that

3 recommended by adult guidelines for performance enhancement (Jeukendrup 2004).

4 However, a significant ergogenic effect was still reported. This evidence, from two studies using the same exercise protocol and $\mathrm{CHO}$ concentration $([\mathrm{CHO}])$, and similar participants,

6 suggests that $\mathrm{CHO}_{\text {exo }}$ requirements are different for adolescents than adults, with perhaps a

7 lower $\mathrm{CHO}$ intake able to promote significant exercise enhancement (Phillips et al

8 unpublished data).

10 Body mass loss and sweat rate

12 The non-significant between-trials difference in BM loss in the current study is in agreement with Phillips et al (2010) and previous adult work (Ali et al 2007; Nicholas et al 1995), as is

14 the similar between-trials mean SR (Phillips et al 2010). Together, this data suggests a

15 similar degree of thermal stress, and thermoregulatory ability, between trials. These data

16 represent the only published information currently available on the BM loss and SR of adolescents during simulated team games, therefore further comparative discussion is not

18 possible.

19

$20 \quad$ Blinding

21

22 Analysis of the solution responses in the $\mathrm{CHO}$ trial demonstrated that the blinding procedures used in this study were successful. However, as discussed by Phillips et al (2010), it may be inappropriate to evaluate the success of blinding procedures simply by comparing them to chance (Boutron et al 2005). It appears that exercise did not provide any cues enabling 
1 participants to more accurately choose both gels post-exercise. Analysis of the individual

2 trials suggests that exercise made it easier for participants to recognise the PLA gel and

3 harder to recognise the $\mathrm{CHO}$ gel, although the response rate was near to the $50 \%$

4 correct/incorrect response rate that could be expected to occur by chance alone (Boutron et al $52005)$

6

\section{Conclusion}

9 Ingestion of a $\mathrm{CHO}$ gel immediately before, and during, a simulated team games protocol

10 significantly improves the intermittent endurance capacity of 12-14 year old team games

11 players. Carbohydrate gel ingestion does not significantly influence the sprint performance

12 or physiological responses of adolescents during simulated team games.

13

14

15

16 


\section{Acknowledgements}

2

3 The authors gratefully acknowledge the support of High5 Ltd, Bardon, Leicestershire, UK for

4 the supply of CHO and PLA gels and drink bottles to enable completion of this study.

5

6

8

10

11

12

13

14

15

16

17

18

19

20

21 


\section{Conflict of Interest}

2

3 The authors declare they have no conflict of interest regarding this study.

4

5

6

7

8

9

10

11

12

13

14

15

16

17

18

19

20

21

22

23

24 


\section{Ethical Declaration}

2

3 The authors confirm that the conduct of this study complied fully with current Scottish law,

4 and with the full ethical approval of the University of Edinburgh, Moray House School of

5 Education Ethics Committee.

6

8

9

10

11

12

13

14

15

16

17

18

19

20

21

22

23

24

25 
3 Ali A, Gant N, Foskett A, Moss C, Lynch C (2009) The modified Loughborough Intermittent

4 Shuttle Test (LIST): A performance tool for use with games players [abstract]. $14^{\text {th }}$ Annual

5 Congress of the European College of Sports Science; June 24-27; Oslo, Norway: 608.

6

7 Ali A, Williams C, Nicholas W, Foskett A (2007) The influence of carbohydrate-electrolyte 8 ingestion on soccer skill performance. Med Sci Sports Exerc 39: 1969-1976.

9

10 Armstrong N (Ed) (2007) Paediatric Exercise Physiology. Churchill Livingstone Elsevier,

11 Philadelphia.

13 Aucouturier J, Baker JS, Duché P (2008) Fat and carbohydrate metabolism during

14 submaximal exercise in children. Sports Med 38: 213-238.

15

16 Bangsbo J (1994) The physiology of soccer with special reference to intense intermittent

17 exercise. Acta Physiol Scand (Suppl) 619: 1-155.

18

19 Baxter-Jones ADG, Eisenmann JC, Sherer LB (2005) Controlling for maturation in pediatric 20 exercise science. Pediatr Exerc Sci 17: 18-30.

21

Boutron I, Estellat C, Ravaud P (2005) A review of blinding in randomised controlled trials

23 found results inconsistent and questionable. J Clin Epidemiol 58: 1220-1226. 
1 Burke LM, Wood C, Pyne DB, Telford RD, Saunders PU (2005) Effect of carbohydrate

2 intake on half-marathon performance of well-trained runners. Int J Sport Nutr Exerc Metab

3 15: 573-589.

4

5 Campbell C, Prince D, Braun M, Applegate E, Casazza GA (2008) Carbohydrate-supplement

6 form and exercise performance. Int J Sport Nutr Exerc Metab. 18: 179-190.

7

8 Carling C, Bloomfield J, Nelsen L, Reilly T (2008) The role of motion analysis in elite

9 soccer: contemporary performance measurement techniques and work rate data. Sports Med

$10 \quad 38: 839-862$.

11

12 Crouter S, Foster C, Esten P, Brice G, Porcari JP (2001) Comparison of incremental treadmill

13 exercise and free range running. Med Sci Sports Exerc 33: 644-647.

14

15 Earnest CP, Lancaster SL, Rasmussen CJ, Kerksick CM, Lucia A, Greenwood MC, Almada

16 AL, Cowan PA, Kreider RB (2004) Low vs. high glycemic index carbohydrate gel ingestion

17 during simulated 64-km cycling time trial performance. J Strength Cond Res 18: 466-472.

18

19 Edwards AM, Mann ME, Marfell-Jones MJ, Rankin DM, Noakes TD, Shillington DP (2007)

20 Influence of moderate dehydration on soccer performance: physiological responses to $45 \mathrm{~min}$

21 of outdoor match-play and the immediate subsequent performance of sport-specific and

22 mental concentration tests. Br J Sports Med 41: 385-391.

23

24 Field A (2005) Discovering Statistics Using SPSS (2 ${ }^{\text {nd }}$ ed). Sage, London. 
1 Foskett A, Williams C, Boobis L, Tsintzas K (2008) Carbohydrate availability and muscle

2 energy metabolism during intermittent running. Med Sci Sports Exerc 40: 96-103.

3

4 Havemann L, Goedecke JH (2008) Nutritional practices of male cyclists before and during an

5 ultraendurance event. Int J Sport Nutr Exerc Metab 18: 551-566.

6

7 Hopkins WG (2006) A Scale of Magnitudes for Effect Statistics. Sportscience.

8 http://sportsci.org/resource/stats/. Accessed 19 January 2011.

9

10 Jeukendrup A (2004) Carbohydrate intake during exercise and performance. Nutrition 20:

11 669-677.

12

13 Lavcanska V, Taylor NF \& Schache AG (2005) Familiarization to treadmill running in young

14 unimpaired adults. Hum Mov Sci 24: 544-557.

15

16 Marino FE, Lambert MI, Noakes TD (2004) Superior performance of African runners in

17 warm humid but not in cool environmental conditions. J Appl Physiol 96: 124-130.

18

19 Mirwald RL, Baxter-Jones ADG, Bailey DA, Beunen GP (2002) An assessment of maturity

20 from anthropometric measurements. Med Sci Sports Exerc 34: 689-694.

21

22 Nicholas CW, Williams C, Lakomy HKA, Phillips G, Nowitz A (1995) Influence of

23 ingesting a carbohydrate-electrolyte solution on endurance capacity during intermittent, high-

24 intensity shuttle running. J Sports Sci 13: 283-290.

25 
1 Noakes T, Rehrer N, Maughan R (1991) The importance of volume in regulating gastric

2 emptying. Med Sci Sports Exerc 23: 307-313.

3

4 Patterson SD, Gray SC (2007) Carbohydrate-gel supplementation and endurance performance

5 during intermittent high-intensity shuttle running. Int J Sport Nutr Exerc Metab 17: 445-55.

6

7 Pfeiffer B, Stellingwerff T, Zaltas E, Jeukendrup AE (2010) Carbohydrate oxidation from a

8 carbohydrate gel compared to a drink during exercise. Med Sci Sports Exerc 42: 2038-2045.

9

10 Phillips SM, Turner AP, Gray S, Sanderson MF, Sproule J (2010) Ingesting a 6\%

11 carbohydrate-electrolyte solution improves endurance capacity, but not sprint performance,

12 during intermittent, high-intensity shuttle running in adolescent team games players aged 12-

1314 years. Eur J Appl Physiol 109: 811-821.

14

15 Riddell MC (2008) The endocrine response and substrate utilization during exercise in

16 children and adolescents. J Appl Physiol 105: 725-733.

17

18 Roemmich JN, Barkley JE, Epstein LH, Lobarinas CL, White TM, Foster JH (2006) Validity

19 of PCERT and OMNI walk/run ratings of perceived exertion. Med Sci Sports Exerc 38:

$20 \quad 1014-1019$

21

22 Rosenthal R (1991) Meta-analytic Procedures for Social Research (revised). Hillsdale, New 23 Jersey: Erlbaum.

25 Rosnow RL, Rosenthal R (2005) Beginning Behavioural Research: A Conceptual Primer (5 ${ }^{\text {th }}$

26 ed). Pearson/Prentice Hall, New Jersey. 
2 Sandercock G, Voss C, Gladwell V (2008) Twenty-metre shuttle run test performance of

3 English children aged 11 - 15 years in 2007: comparisons with international standards. J

4 Sports Sci 26: 953-957.

5

6 Timmons BW, Bar-or O, Riddell MC (2003) Oxidation rate of exogenous carbohydrate

7 during exercise is higher in boys than in men. J Appl Physiol 94: 278-284.

8

9 Timmons BW, Bar-Or O, Riddell MC (2007) Influence of age and pubertal status on

10 substrate utilization during exercise with and without carbohydrate intake in healthy boys.

11 Appl Physiol Nutr Metab 32: 416-425.

12

13 Welsh RS, Davis JM, Burke JR, Williams HG (2002) Carbohydrates and physical/mental

14 performance during intermittent exercise to fatigue. Med Sci Sports Exerc 34: 723-731.

15

16

17

18

19

20

21 
3 Table 1 Mean heart rate (beats per min) and mean ratings of perceived exertion during part

4 A of the LIST, and mean peak HR and mean ratings of perceived exertion at exhaustion in

5 part B for both trials.

\section{Period of the LIST}

\begin{tabular}{|c|c|}
\hline & Block 2 \\
\hline
\end{tabular}

\section{Mean heart rate}

(beats per min)

$\begin{array}{lrrrrr}\text { CHO } & 158 \pm 7 & 163 \pm 7 * * * & 164 \pm 7 & 163 \pm 6 & 187 \pm 5 \\ \text { PLA } & 158 \pm 9 & 162 \pm 10^{* * * *} & 163 \pm 9 & 162 \pm 9 & 185 \pm 5\end{array}$

Mean ratings of perceived exertion

$\begin{array}{llllll}\text { CHO } & 5.1 \pm 1.4 & 6.4 \pm 1.1^{* * *} & 7.2 \pm 0.6 * * * & 8.1 \pm 0.5 * * * & 9.4 \pm 0.5 \\ \text { PLA } & 5.0 \pm 1.1 & 6.3 \pm 0.9^{* * *} & 7.3 \pm 1.0^{* * *} & 8.2 \pm 0.8^{* * *} & 9.4 \pm 0.50\end{array}$

7 mean RPE)

$8 \mathrm{CHO}=$ carbohydrate trial; $\mathrm{PLA}=$ placebo trial

$9 * * *$ significantly greater than previous block, $P<0.001$

10 
1 Table 2 Mean gut fullness and gastric discomfort ratings during part A of the LIST, and at

2 exhaustion in part $\mathrm{B}$, for both trials.

\begin{tabular}{|c|c|c|c|c|c|}
\hline \multicolumn{6}{|c|}{ Period of the LIST } \\
\hline & Block 1 & Block 2 & Block 3 & Block 4 & Exhaustion \\
\hline \multicolumn{6}{|c|}{$\begin{array}{l}\text { Mean gut fullness } \\
\text { ratings }\end{array}$} \\
\hline $\mathrm{CHO}$ & $4.3 \pm 1.9$ & $4.4 \pm 1.9$ & $5.2 \pm 1.6 \dagger$ & $5.2 \pm 1.8$ & $5.3 \pm 1.8$ \\
\hline PLA & $3.4 \pm 1.3$ & $4.0 \pm 1.1$ & $4.5 \pm 1.4 \dagger$ & $4.5 \pm 1.5$ & $4.7 \pm 1.3$ \\
\hline \multicolumn{6}{|c|}{$\begin{array}{l}\text { Mean gastric } \\
\text { discomfort ratings }\end{array}$} \\
\hline $\mathrm{CHO}$ & $3.0 \pm 1.7$ & $3.5 \pm 2.0 * *$ & $4.5 \pm 2.0 * *$ & $4.5 \pm 2.3$ & $5.5 \pm 2.3$ \\
\hline PLA & $2.8 \pm 1.6$ & $3.9 \pm 1.9 * *$ & $4.3 \pm 2.5^{* *}$ & $4.3 \pm 2.3$ & $5.3 \pm 2.0$ \\
\hline
\end{tabular}

3 Data are mean $\pm \mathrm{SD}(n=11)$

$4 \mathrm{CHO}=$ carbohydrate trial; $\mathrm{PLA}=$ placebo trial

$5 \dagger$ significantly greater than previous block, $\mathrm{P}<0.01$

$6 * *$ significantly greater than previous block, $P<0.05$

7

8 
1 Table 3 Mean ambient temperature $\left({ }^{\circ} \mathrm{C}\right)$ and relative humidity $(\%)$ immediately before, and

2 during, part A of the LIST in both trials.

\begin{tabular}{|c|c|c|c|c|c|}
\hline \multicolumn{6}{|c|}{ Period of the LIST } \\
\hline & Pre-exercise & Block 1 & Block 2 & Block 3 & Block 4 \\
\hline \multicolumn{6}{|c|}{$\begin{array}{l}\text { Mean ambient } \\
\text { temperature }\left({ }^{\circ} \mathrm{C}\right)\end{array}$} \\
\hline $\mathrm{CHO}$ & $18.5 \pm 1.2$ & $18.5 \pm 1.2$ & $18.5 \pm 1.3$ & $18.5 \pm 1.3$ & $18.5 \pm 1.3$ \\
\hline PLA & $18.8 \pm 1.1$ & $18.9 \pm 1.1$ & $18.9 \pm 1.1$ & $18.9 \pm 1.1$ & $18.9 \pm 1.1$ \\
\hline \multicolumn{6}{|c|}{$\begin{array}{l}\text { Mean relative } \\
\text { humidity }(\%)\end{array}$} \\
\hline $\mathrm{CHO}$ & $39.5 \pm 8.0$ & $39.5 \pm 8.1$ & $39.4 \pm 8.2$ & $39.5 \pm 8.6$ & $39.2 \pm 8.5$ \\
\hline PLA & $45.6 \pm 7.5$ & $44.8 \pm 7.3$ & $44.7 \pm 7.1$ & $44.6 \pm 7.3$ & $44.6 \pm 7.3$ \\
\hline
\end{tabular}

3 Data are mean $\pm \mathrm{SD}(n=11)$

$4 \mathrm{CHO}=$ carbohydrate trial; PLA $=$ placebo trial

$5 \quad$ A significant interaction effect was reported for mean ambient temperature $(P<0.05)$ 


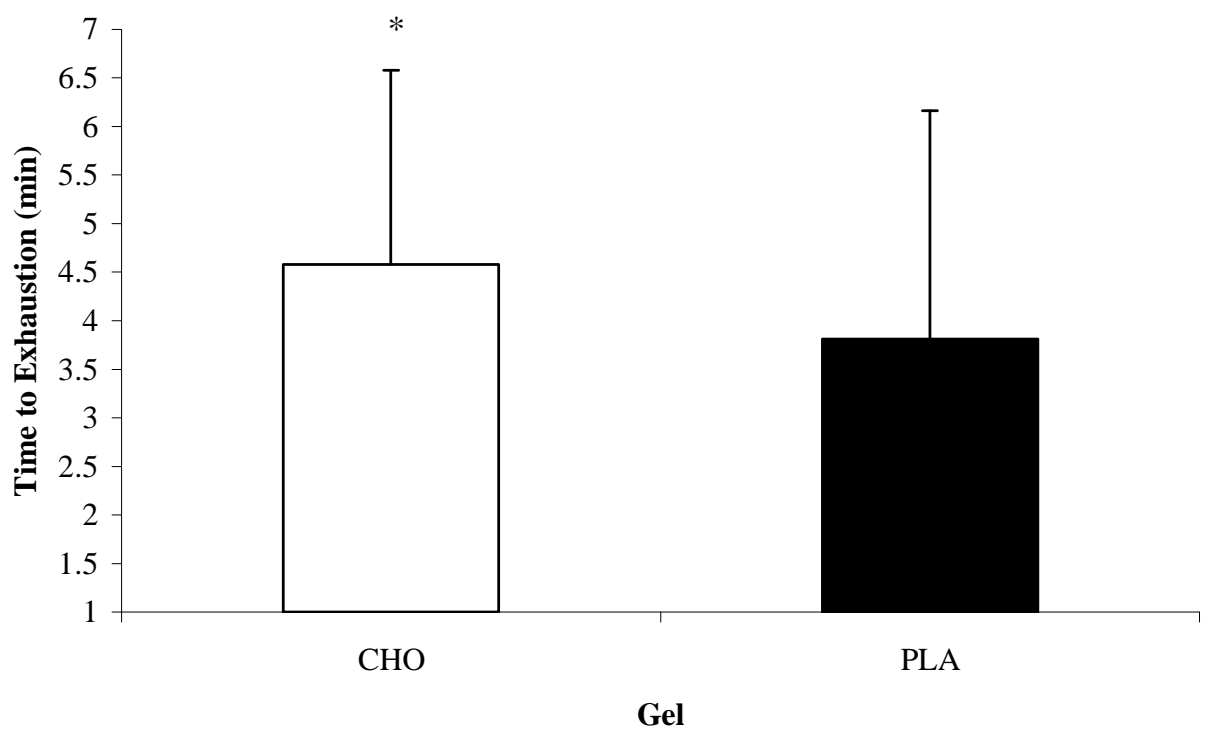

1

3 Figure 1 Time to exhaustion (min) during part $\mathrm{B}$ of the LIST in the CHO and PLA trial.

Deleted: Figure Captions

4 * significantly greater than the PLA trial, $P<0.05 .(n=11)$. 


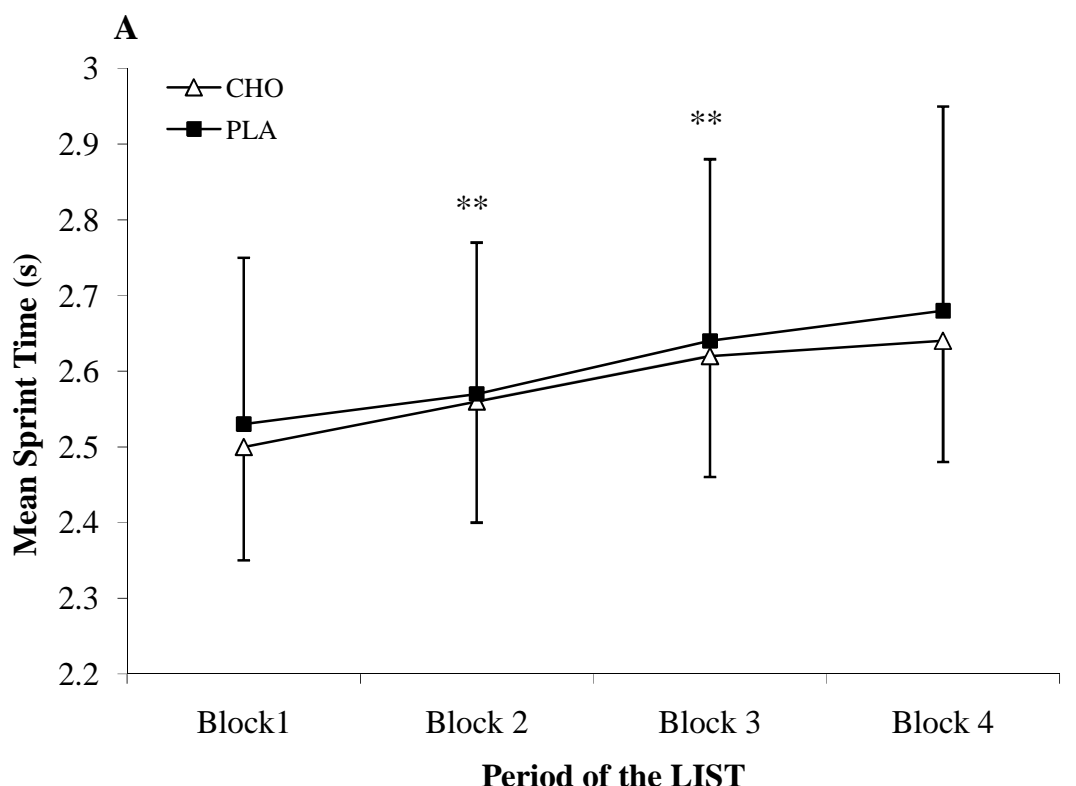

3
4

Period of the LIST

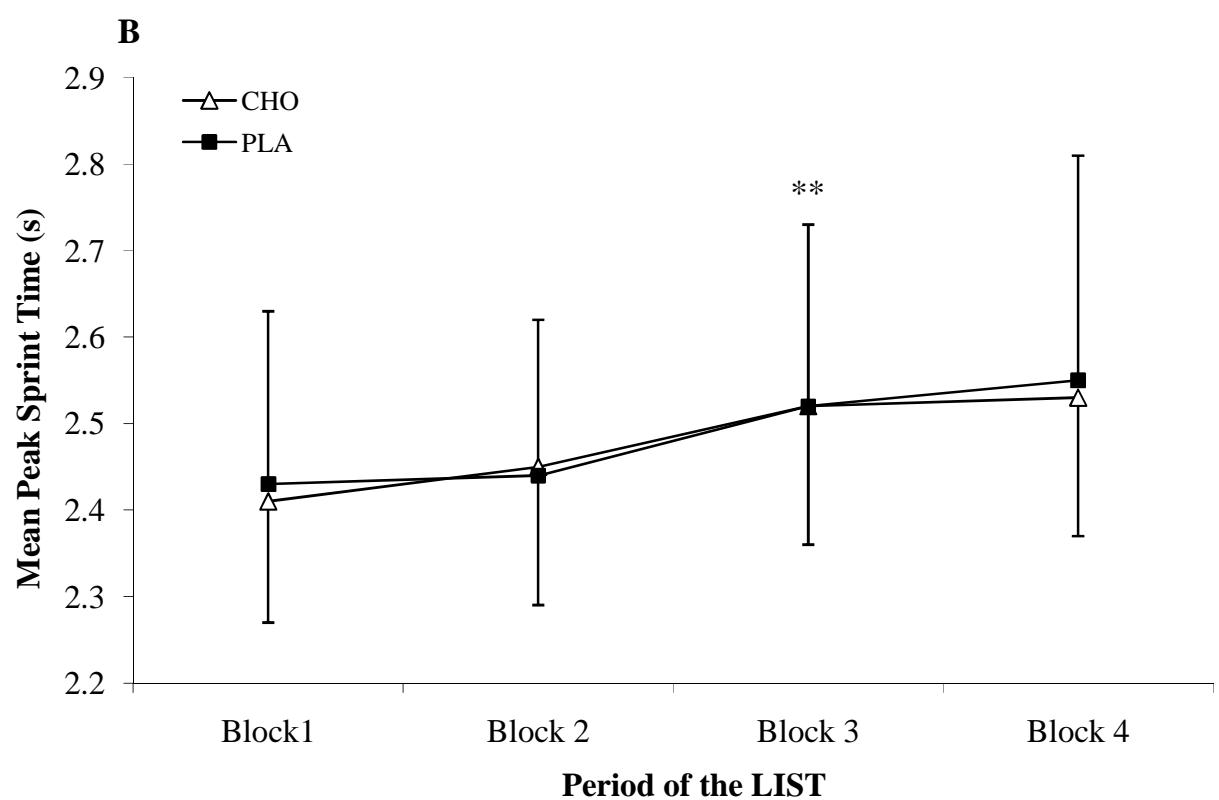

5
6

7 Figure 2 Mean sprint time (s, A) and mean peak sprint time (s, B) during part A of the LIST

8 for both trials. ** significantly greater than previous block, $P<0.05$. $(n=11)$. 